Moqbil Sara, Niedobylski Sylwiusz, Laszczak Katarzyna, Warchol Konrad, Mikos Eryk. The gut microbiota and mental health. Journal of Education, Health and Sport. 2021;11(9):304-309. eISSN 2391-8306. DOI http://dx.doi.org/10.12775/JEHS.2021.11.09.037 https://apcz.umk.pl/JEHS/article/view/JEHS.2021.11.09.037 https://zenodo.org/record/5515293

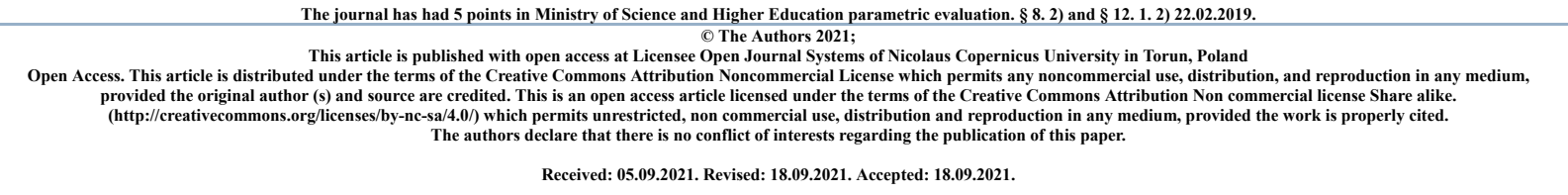

\title{
The gut microbiota and mental health
}

\author{
Sara Moqbil; 0000-0003-1230-1444 \\ Sylwiusz Niedobyski; 0000-0001-7266-623X \\ Katarzyna Laszczak;_0000-0002-5084-0273 \\ Konrad Warchol; 0000-0001-9467-680X \\ Eryk Mikos; 0000-0003-0507-2882
}

Sara Moqbil, Sylwiusz Niedobylski, Katarzyna Laszczak, Konrad Warchoł, Eryk Mikos

\begin{abstract}
INTRODUCTION AND PURPOSE

Recent studies have shown that changes in the microbiome, probiotic and antibiotic supplementation, can significantly modulate various forms of neuropsychiatric disorders such as depression, anxiety and stress-related disorders. There is growing body of evidence pointing to a bidirectional correlation along the brain-gut microbiota line. This axis is connected through endocrine, immune and neuronal pathways. The nerves that make up the enteric nervous system transmit modifications occurring in the gastrointestinal tract and through the vagus nerve to the central nervous system (1). The main purpose of this review is to update recent information on the correlation between the gut microbiota and mental health.
\end{abstract}

\section{STATE OF KNOWLEDGE}

Interactions between the gastrointestinal system and brain function have become an important field of psychiatric research in recent years. Probiotics are thought to be a potentially valuable player in the treatment of many neuropsychiatric disorders. However, the role of specific gut microbiota species in the development of these disorders remains unclear.

\section{CONCLUSIONS}

Increasing knowledge of the correlation between gut microbiota and mental health may improve the quality of treatment for patients with neuropsychiatric conditions. Further research on larger groups is needed to assess whether probiotics can modify altered psychological well-being and be integrated into current, conventional treatments. 
Key words: gut microbiota, mental health

\section{INTRODUCTION}

Proper gut function is determined by the healthy functioning of the central nervous system. Simultaneously, by sending signals to the brain, immune factors and hormones released from the gut affect brain function. New researches indicate that gut bacteria can activate neuronal pathways and central nervous system (CNS) signaling systems. Since this correlation was discovered, many studies have focused on the use of probiotics in patients with neuropsychiatric disorders. Understanding the gut microbiota-brain axis may be crucial in uncovering the exact mechanisms of depression, stress, anxiety-related behaviors and the human body's resistance to them.

\section{DEPRESSION}

A study on rats that were separated from their mothers during the neonatal period showed that a decrease in depressive symptoms was observed after administration of the probiotic Bifidobacterium infantism. The same behavioral effect was observed after administration of citalopram [2]. The results of 2019 study indicate that the gut microbiota may be contributing in the pathogenesis of major depressive disorder (MDD. Group consisted of thirty one patients with MDD, thirty patients with bipolar disorder with current major depressive episode (BPD) and thirty healthy controls. They have been tested using faecal samples analyzed by shotgun metagenomics sequencing. The genera like - Bacteroides, Clostridium, Bifidobacterium, Oscillibacter and Streptococcus - were significantly decreased in healthy individuals compared to MDD and BPD patients. Additionally, the MDD group showed significantly increased Firmicutes and Actinobacteria and decreased Bacteroidetes compared to the BPD patients. Differences in gut microbiota between MDD and BPD patients suggest that specific bacterial species could potentially serve as biomarkers for these disorders [3]. Shaohua $\mathrm{Hu}$ et al. conducted a study revealing that untreated depressed patients with bipolar disorder (BD) demonstrate changes in the gut microbiota [4]. 140 samples from $72 \mathrm{BD}$ patients and 45 healthy controls were tested. A definite enrichment of Firmicutes phylum, Roseburia, Faecalibacterium and Coprococcus genera was found in healthy individuals, while Bacteroidetes phylum, Parabacteroides, Bacteroides and Halomonas genera were more abundant in BD patients. Additionally, it was concluded that BD treatment with quetapine also significantly affects the diversification of the gut microbiome - treated patients have higher levels of butyrate-producing bacteria. Similar findings were presented in a study on 54 patients, 27 of whom suffered from major depressive disorder (MDD). Analysis of fecal samples by $16 \mathrm{~S}$ rRNA sequencing showed that MDD patients had a significantly lower level of microbiome diversity compared to healthy controls, with Firmicutes being the most reduced phylum [5]. The possible involvement of the microbiome in the pathophysiology of depression has led researchers to study the effects of taking probiotics in patients with 
depression. The 8- week prospective open-label trial of 40 patients with treatment-resistant major depressive disorder (TRD) involved treatment with Clostridium butyricum MIYAIRI 588 (CBM588) in combination with conventional antidepressants (flvoxamine, paroxetine, escitalopram, duroxetine, and sertraline). The results confirmed the effectiveness and tolerability of patients on this treatment, with $70 \%$ of patients responding to it. Additionally, a remission rate of $35 \%$ was achieved [6]. As one of many factors, detrimental kynurenine catabolites are responsible for contributing to the pathophysiology of depression. A doubleblind, randomized, placebo controlled study examined the levels of kynurenine and its metabolites after treatment with the probiotic Lactobacillus Plantarum 299v (LP299v). The improvements in cognitive function in depressed patients and significant reductions in kynurenine levels were observed [7]. A randomized clinical trial conducted on patients with MDD compared the effects of probiotic and prebiotic supplementation. The effects of treatment were tested using the Beck Depression Inventory (BDI) score. The most significant decrease in the index (17.39-9.1) was observed in 28 patients in the probiotic group compared to the placebo group and the prebiotic group, in which no significant effect was observed (19.72-14.14) [8]. A randomized, triple-blind, placebo-controlled trial of 71 patients assigned to a probiotic and a placebo group found that - although probiotics did not significantly affect the microbiota of depressed patients - they were associated with the depression susceptibility variable. Ruminococcus gnavus significantly and positively correlate (0.37) with DASS depression score. The coccus was significantly more abundant in depressed patients $(72 \%)$, in the healthy group its abundance was $25 \%$. A higher relative abundance characterized the group of patients in the severe range of depression according to the BDI scale [9].

\section{STRESS AND ANXIETY DISORDER}

Accumulating evidence indicates links between disordered gut microbiome composition and stress or anxiety behaviors. Studies on rats have shown that neonatal stress affects the diversity and composition of the gut microbiota, which in turn may contribute to changes in stress-related behaviors [10]. The involvement of gastrointestinal bacteria in the activation of stress circuits has been demonstrated by studies using oral administration of Citrobacter rodentium and Campylobacter jejuni. The vagal pathways are the connecting axis $[11,12]$. Abnormalities in GABA receptor expression underlie the pathogenesis of anxiety and depression, much of which occurs concurrently with gut dysfunction. The correlation of gastrointestinal bacteria with GABA neurotransmitter expression was demonstrated in a study conducted on mice exposed to chronic treatment with L. rhamnosus (JB-1). Treatment was shown to induce region-dependent changes in GABA(B1b) mRNA expression in the brain. Compared to control-fed mice, expression increased in cortical regions (cingulate cortex and prelimbic cortex), whereas it decreased in the hippocampus, amygdale and locus coeruleus [13]. In another study, rats were subjected to a 4-week chronic mild stress (CMS) model to investigate the potential effects of Lactobacillus fermentum PS150 (PS150) on anxiety and depressive behaviors. PS150 supplementation has resulted in preventing serotonin reduction and neurodegeneration in the brain [14]. Results of the randomized, double-blind, placebocontrolled study on 60 Japanese medical students exposed to taking heat-inactivated Lactobacillus gasseri CP2305 once daily for 24 weeks revealed that this treatment significantly reduced anxiety and sleep disturbances. These factors were assessed by the Spielberger Anxiety Questionnaire and the Pittsburgh Sleep Quality Index. Additionally, administration of CP2305 resulted in an upregulation in the composition of the gut microbiota disrupted under stress (stress factors cause a decrease in Bifidobacterium spp. and an increase in Streptococcus spp.) [15]. Yi-huan Chen et al. examined microbiome composition in 36 patients with active generalized anxiety disorder (GAD) compared with 24 healthy controls. 
Faecal samples were analyzed by sequencing the 16S gene and anxiety levels were assessed using the Hamilton Anxiety Rating Scale and the Anxiety Self-Assessment Scale. Lower abundance of Firmicutes and Tenericutes was observed in patients with active GAD. Bacteroides and Escherichia-Shigella abundance showed a positive correlation with anxiety severity, in contrast to Eubacterium_coprostanoligenes_group, Ruminococcaceae_UCG-014, and Prevotella_9, which correlate positively with anxiety reduction [16]. The effect of taking probiotics on stress relief was examined in a 12-week randomized, double-blind, placebocontrolled study. A group consisting of 103 middle-aged stressed adults took the probiotic Lactobacillus plantarum P8. Using the DASS-42 questionnaire, it was presented that P8 supplementation led to lower anxiety and stress scores. It also had a positive effect on cognitive traits and memory [17].

\section{CONCLUSIONS}

The reviewed studies provide valuable insight into the gut microbiota-brain axis. The discovery of this bidirectional relationship provides a foundation for understanding the impact of microbiota diversity and probiotics on central nervous system functioning. Thorough studies on larger groups will accurately determine the role of probiotics in the treatment of numerous neuropsychiatric disorders and may improve the quality of life of these patients.

\section{References}

[1] Furness JB, Kunze WA, Clerc N. Nutrient tasting and signaling mechanisms in the gut. II. The intestine as a sensory organ: neural, endocrine, and immune responses. Am J Physiol. 1999 Nov;277(5):G922-8. doi: 10.1152/ajpgi.1999.277.5.G922. PMID: 10564096.

[2] Desbonnet L, Garrett L, Clarke G, Kiely B, Cryan JF, Dinan TG. Effects of the probiotic Bifidobacterium infantis in the maternal separation model of depression. Neuroscience. 2010 Nov 10;170(4):1179-88. doi: 10.1016/j.neuroscience.2010.08.005. Epub 2010 Aug 6. PMID: 20696216.

[3] Rong H, Xie XH, Zhao J, Lai WT, Wang MB, Xu D, Liu YH, Guo YY, Xu SX, Deng WF, Yang QF, Xiao L, Zhang YL, He FS, Wang S, Liu TB. Similarly in depression, nuances of gut microbiota: Evidences from a shotgun metagenomics sequencing study on major depressive disorder versus bipolar disorder with current major depressive episode patients. J Psychiatr Res. 2019 Jun;113:90-99. doi: 10.1016/j.jpsychires.2019.03.017. Epub 2019 Mar 21. PMID: 30927646.

[4] Hu S, Li A, Huang T, Lai J, Li J, Sublette ME, Lu H, Lu Q, Du Y, Hu Z, Ng CH, Zhang H, Lu J, Mou T, Lu S, Wang D, Duan J, Hu J, Huang M, Wei N, Zhou W, Ruan L, Li MD, Xu Y. Gut Microbiota Changes in Patients with Bipolar Depression. Adv Sci (Weinh). 2019 May 15;6(14):1900752. doi: 10.1002/advs.201900752. PMID: 31380217; PMCID: PMC6662053.

[5] Huang Y, Shi X, Li Z, Shen Y, Shi X, Wang L, Li G, Yuan Y, Wang J, Zhang Y, Zhao L, Zhang M, Kang Y, Liang Y. Possible association of Firmicutes in the gut microbiota of patients with major depressive disorder. Neuropsychiatr Dis Treat. 2018 Dec 3;14:3329-3337. doi: 10.2147/NDT.S188340. PMID: 30584306; PMCID: PMC6284853. 
[6] Miyaoka T, Kanayama M, Wake R, Hashioka S, Hayashida M, Nagahama M, Okazaki S, Yamashita S, Miura S, Miki H, Matsuda H, Koike M, Izuhara M, Araki T, Tsuchie K, Azis IA, Arauchi R, Abdullah RA, Oh-Nishi A, Horiguchi J. Clostridium butyricum MIYAIRI 588 as Adjunctive Therapy for Treatment-Resistant Major Depressive Disorder: A Prospective Open-Label Trial. Clin Neuropharmacol. 2018 Sep/Oct;41(5):151-155. doi: 10.1097/WNF.0000000000000299. PMID: 30234616.

[7] Miyaoka, Tsuyoshi MD, PhD; Kanayama, Misako MD; Wake, Rei MD, PhD; Hashioka, Sadayuki MD, PhD; Hayashida, Maiko MD, PhD; Nagahama, Michiharu MD; Okazaki, Shihoh MD; Yamashita, Satoko MD; Miura, Shoko MD; Miki, Hiroyuki MD; Matsuda, Hiroyuki MD; Koike, Masahiro MD; Izuhara, Muneto MD; Araki, Tomoko MSc; Tsuchie, Keiko MSc; Azis, Ilhamuddin Abdul MD; Arauchi, Ryosuke MSc; Abdullah, Rostia Arianna MD; Oh-Nishi, Arata PhD; Horiguchi, Jun MD, PhD Clostridium butyricum MIYAIRI 588 as Adjunctive Therapy for Treatment-Resistant Major Depressive Disorder: A Prospective Open-Label Trial, Clinical Neuropharmacology: 9/10 2018 - Volume 41 - Issue 5 - p 151-155 doi: 10.1097/WNF.0000000000000299

[8] Asma Kazemi, Ahmad Ali Noorbala, Kamal Azam, Mohammad Hadi Eskandari, Kurosh Djafarian, Effect of probiotic and prebiotic vs placebo on psychological outcomes in patients with major depressive disorder: A randomized clinical trial, Clinical Nutrition, Volume 38, Issue 2, 2019,Pages 522-528,ISSN 0261-5614, https://doi.org/10.1016/j.clnu.2018.04.010.

[9] Chahwan B, Kwan S, Isik A, van Hemert S, Burke C, Roberts L. Gut feelings: A randomised, triple-blind, placebo-controlled trial of probiotics for depressive symptoms. J Affect Disord. 2019 Jun 15;253:317-326. doi: 10.1016/j.jad.2019.04.097. Epub 2019 May 9. PMID: 31078831.

[10] O’Mahony, S.M. et al. (2009) Early life stress alters behavior, immunity, and microbiota in rats: implications for irritable bowel syndrome and psychiatric illnesses. Biol. Psychiatry $65,263-267$

[11] Goehler, L.E. et al. (2008) Campylobacter jejuni infection increases anxiety-like behavior in the holeboard: possible anatomical substrates for viscerosensory modulation of exploratory behavior. Brain Behav. Immun. 22, 354-366

[12] Lyte, M. et al. (2006) Induction of anxiety-like behavior in mice during the initial stages of infection with the agent of murine colonic hyperplasia Citrobacter rodentium. Physiol. Behav. 89, 350-357

[13] Bravo JA, Forsythe P, Chew MV, Escaravage E, Savignac HM, Dinan TG, Bienenstock J, Cryan JF. Ingestion of Lactobacillus strain regulates emotional behavior and central GABA receptor expression in a mouse via the vagus nerve. Proc Natl Acad Sci U S A. 2011 Sep 20;108(38):16050-5. doi: 10.1073/pnas.1102999108. Epub 2011 Aug 29. PMID: 21876150; PMCID: PMC3179073.

[14] Yen-Wenn Liu, Jia Sin Ong, Chee Yuen Gan, Boon Yin Khoo, Sawibah Yahaya, Sy Bing Choi, Wai Yee Low, Ying-Chieh Tsai, Min Tze Liong, Lactobacillus fermentum PS150 showed psychotropic properties by altering serotonergic pathway during stress, Journal of Functional Foods, Volume 59, 2019, Pages 352-361, ISSN 1756-4646, https://doi.org/10.1016/j.jff.2019.05.043. 
[15] Nishida, K.; Sawada, D.; Kuwano, Y.; Tanaka, H.; Rokutan, K. Health Benefits of Lactobacillus gasseri CP2305 Tablets in Young Adults Exposed to Chronic Stress: A Randomized, Double-Blind, Placebo-Controlled Study. Nutrients 2019, 11, 1859. https://doi.org/10.3390/nu11081859

[16] Yi-huan Chen, Jie Bai, Di Wu, Shou-fen Yu, Xiao-ling Qiang, Hua Bai, Hua-ning Wang, Zheng-wu Peng, Association between fecal microbiota and generalized anxiety disorder: Severity and early treatment response, Journal of Affective Disorders, Volume 259, 2019, Pages 56-66, ISSN 0165-0327, https://doi.org/10.1016/j.jad.2019.08.014.

[17] Lee-Ching Lew, Yan-Yan Hor, Nur Asmaa' A. Yusoff, Sy-Bing Choi, Muhamad S.B. Yusoff, Nurhanis S. Roslan, Anisa Ahmad, Jamilah A.M. Mohammad, Mohammad Farris I.L. Abdullah, Norzila Zakaria, Normala Wahid, Zhihong Sun, Lai-Yu Kwok, Heping Zhang, MinTze Liong, Probiotic Lactobacillus plantarum P8 alleviated stress and anxiety while enhancing memory and cognition in stressed adults: A randomised, double-blind, placebocontrolled study, Clinical Nutrition, Volume 38, Issue 5, 2019, Pages 2053-2064, ISSN 02615614, https://doi.org/10.1016/j.clnu.2018.09.010. 